\title{
Infection With the Mycobacterium avium Complex in Patients Without Predisposing Conditons - A Case Report and Literature Review
}

\author{
Andrea Barral Martins ${ }^{1}$, Eliana Dias Matos ${ }^{2,3}$ \\ and Antonio Carlos M. Lemos ${ }^{2,4,5}$
}

\author{
Pneumologist Resident at University Hospital Professor Edgar \\ Santos $^{1}$; Pneumologist at Octávio Mangabeira Specialized \\ Hospital $^{2}$; Assistant Professor at the Department of Medicine, \\ Escola Bahiana de Medicina e Saúde Pública ${ }^{3}$; MD and \\ Associate Professor at the Department of Medicine, Federal \\ University of Bahia (Medical School) ${ }^{4}$; Coordinator of the \\ Pneumology Research Center (NUPEP), Octávio Mangabeira \\ Hospital $^{5}$; Salvador, BA, Brazil
}

\begin{abstract}
Nontuberculous Mycobacteria (NTM), especially Mycobacterium avium-intracellulare complex (MAC), has been considered responsible for human disease, especially in HIV patients. Nevertheless, it has been diagnosed in immunocompetent elderly men, frequently with previous pulmonary disease: chronic obstructive lung disease (COPD), complications of tuberculosis, pulmonary fibrosis and bronchiectasis. We relate the case of a female patient, 51 years old, with continuously acid fast bacilli (AFB) smears and with three previous treatments, which were conducted at the multiresistant tuberculosis (MRTB) service. MAC was identified in the sputum culture, and she received treatment for one year. The posterior sputum exams were negative. The cavity lesions observed in the high-resolution computed tomography (HRCT) were reduced, and some of the nodule lesions became bronchiectasis, even after the end of treatment. We agree with the literature reports that indicate that $\mathrm{MAC}$ is the cause of bronchiectasis. It is necessary to identify the type of mycobacteria in immunocompetent individuals with positive AFB smears that do not become negative with tuberculosis treatment.
\end{abstract}

Key Words: Mycobacterium avium, pulmonary disease, bronchiectasis, immunocompetent.

The Mycobacterium avium-intracellulare complex can cause isolated pulmonary disease and/or systemic disease; the latter is more frequent in patients with HIV immunosuppression [1,2]. In HIV negative patients, MAC has been cultivated from the sputum of patients with previous pulmonary disease, such as bronchiectasis, cystic fibrosis, COPD, and pulmonary fibrosis [1-6]. In this situation, MAC may be only a colonizer of the airways [1,7]. On the other hand, MAC

Received on 21 November 2004; revised 17 February 2005. Address for correspondence: Dr. Antônio Carlos Moreira Lemos. Praça Conselheiro João Alfredo, SN, Pau Miúdo. Salvador-Bahia, Brazil, Zip code: 40.320-350. Phone: 388-2740 Fax: (71) 386-4122/4541. E-mails: acmlemos@uol.com.br / abacontt@ig.com.br.

The Brazilian Journal of Infectious Diseases 2005;9(2):173-179 (C) 2005 by The Brazilian Journal of Infectious Diseases and Contexto Publishing. All rights reserved. pulmonary disease is typically described in elderly white males, smokers and patients with alcohol abuse [8]. It is found in patients with or without previous pulmonary disease [1-6]. It has also been observed in nonsmoking women over age 50, without previous pulmonary disease, in whom the radiographic patterns were preferentially in the lingula and middle lobe $[2,4,5]$. Reich et al. offer the term Lady Windermere's syndrome to describe this type of presentation [5].

We report a case of MAC pulmonary disease in a woman without a predisposing condition or immunosuppression.

\section{Case Report}

A 51-year-old white female, without symptoms, presented a modest lingulare infiltrate in chest 
roentgenograms, which were part of a routine exam in 1995. In 1998, she began to exhibit a chronic cough, fever and weight loss. The sputum smear was positive for acid-fast bacilli, and she started treatment for tuberculosis (TB). Figures 1 and 2 show the roentgenograms and Figures 3 and 4 the high-resolution computed tomographies of the chest, before the use of the first regimen. In these images, we observe a cavitary lesion in the upper lingulare segment (thick black arrows), and small multiple nodules in the middle and lower lobes, and also in the lingulare segment (thin white arrow). In Figure 3, we observe bronchiectasis (thin black arrows) and in Figure 4 we observe groundglass attenuation and air trapping (thick white arrows).

Despite the correct use of medication for six months, she continued symptomatic and with positive AFB smears. A second regimen, rifampin, isomazid, pirazinamid and ethambutol, was initiated, and at the end of the second treatment she was still symptomatic, with positive AFB smears, and the lesions observed in imaging exams became worse (Figures 5, 6 and 7). Progression of the disease was apparent, with a new cavitary lesion in the posterior segment of the right upper lobe (white arrows), and there was persistence of the previously-described findings.

The chest HRCTs at the end of the III regimen (Figures 8 and 9) demonstrate a partial improvement of the cavitary lesion in the posterior segment of the right upper lobe (thick white arrows) and persistence of other findings (thin white arrows). The patient was still symptomatic and had positive AFB smears.

At this point, the patient was classified as a carrier of multiresistant tuberculosis, and she was conducted to a reference service, where a routine culture was taken for mycobacteria; the diagnosis was MAC.

Four more cultures were made, and MAC was isolated in all them.

MAC treatment was applied for 12 months, from September 2001 to August 2002. The drugs were ofloxacin, ethambutol, clofazimine and clarithromycin. She did not use amikacin, because she presented a hearing deterioration with the use of streptomycin during the III regimen; it became necessary to discontinue the drug. At the end of treatment, the patient had few symptoms and negative AFB smears and cultures. She was discharged as she was cured. In January 2003, the results of AFB smears and cultures were negative, and they continued negative in June 2004.

In spite of the negative AFB smears and cultures, the patient presented repeated pulmonary infections, and as demonstrated in the chest HRCT (Figures 1012), there was a progression of the bronchiectasis in the middle lobe and the upper segment of the right lower lobe (white arrows), as well as in the lingulare segment (black arrow). The quantitative pilocarpine iontophoresis sweat test revealed normal chloride levels (31.8 and $30.5 \mathrm{mEq} / \mathrm{l}$ ), rejecting the possibility of cystic fibrosis.

\section{Discussion}

Mycobacterium infections are divided into Mycobacterium tuberculosis and nontuberculous mycobacteriosis (NTM). MAC is the most common among the NTM capable of causing disease in humans [3].

MAC is a free-living organism that can be recovered from natural reservoirs, such as soil, water, domestic and wild animals, milk and other foodstuffs [1,9]. Environmental MAC isolates and clinical isolates belong to different serovars [8]. The strains that cause pulmonary infection in humans are the same that can aerosolize $[1,10,11]$, and they have been recovered from water and piped hot water systems $[1,2,12,13]$. This type of MAC has been demonstrated in hospitals [2], which can explain nosocomial infections in HIV patients [1]. Unlike tuberculosis, it is believed that this infection is acquired by inhalation of environmental MAC in most individuals, and that human-to-human transmission is rare $[1,8,14]$.

Pathogenesis of this disease is poorly understood; according to the literature, the host immune response is very important. Age and preexisting lung disease are major risk factors for active NTM lung disease [8]. There is an interaction between macrophages, lymphocytes and mycobacteria, which is the focal point 
Figure 1.

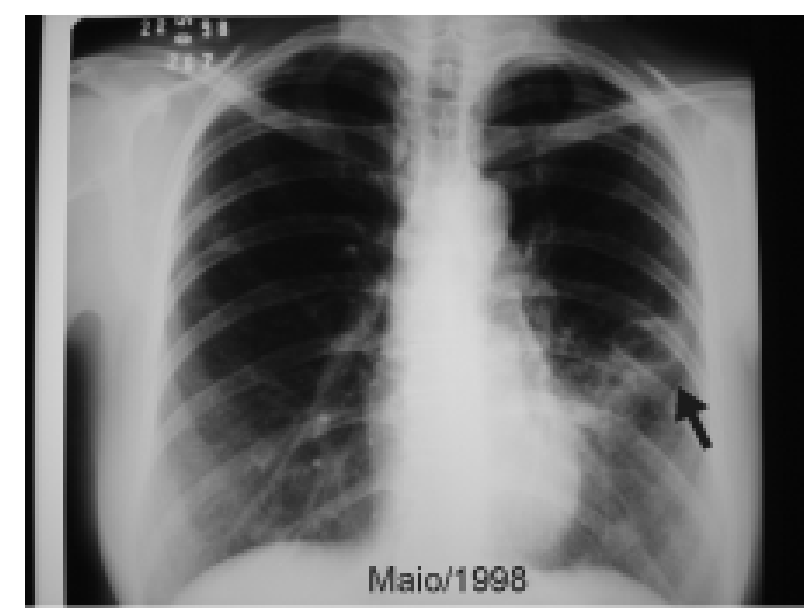

Figure 3.

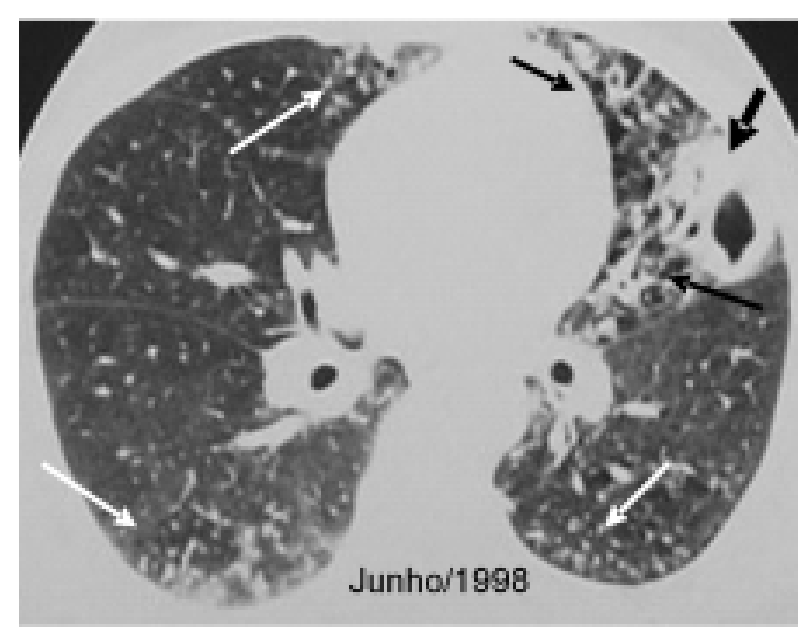

Figure 5.

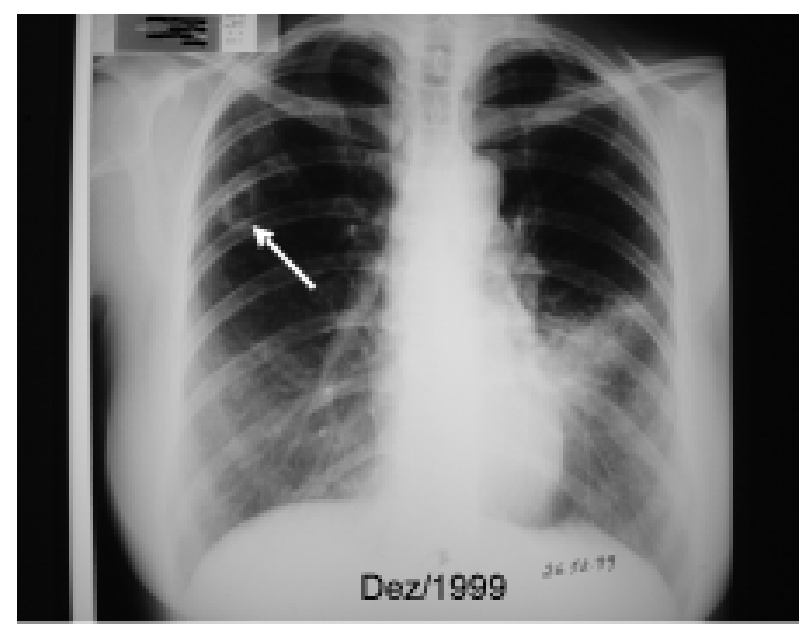

Figure 2.

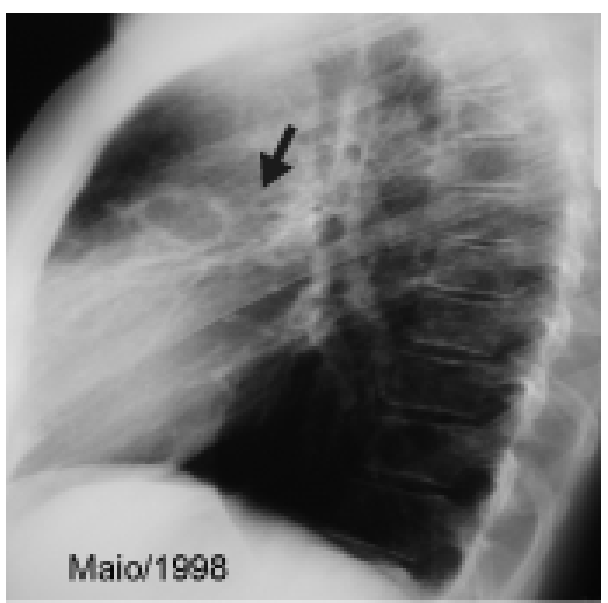

Figure 4.

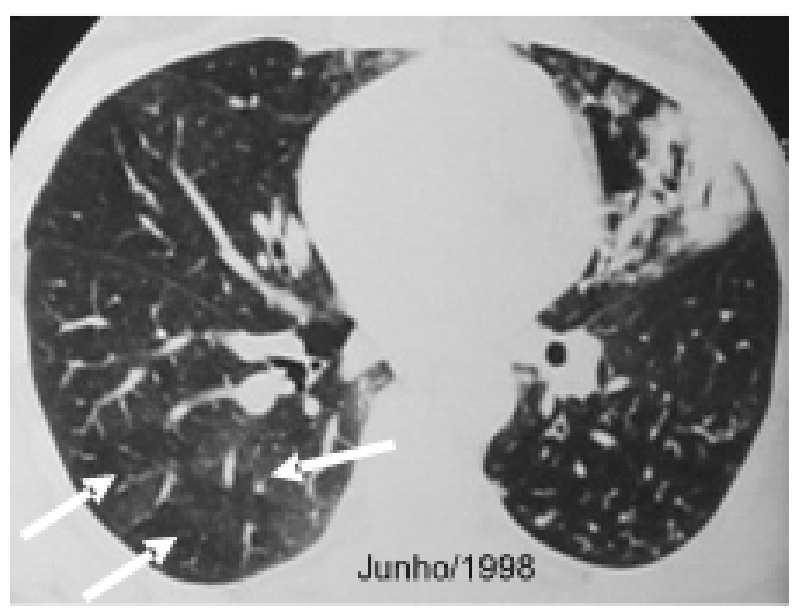

Figure 6.

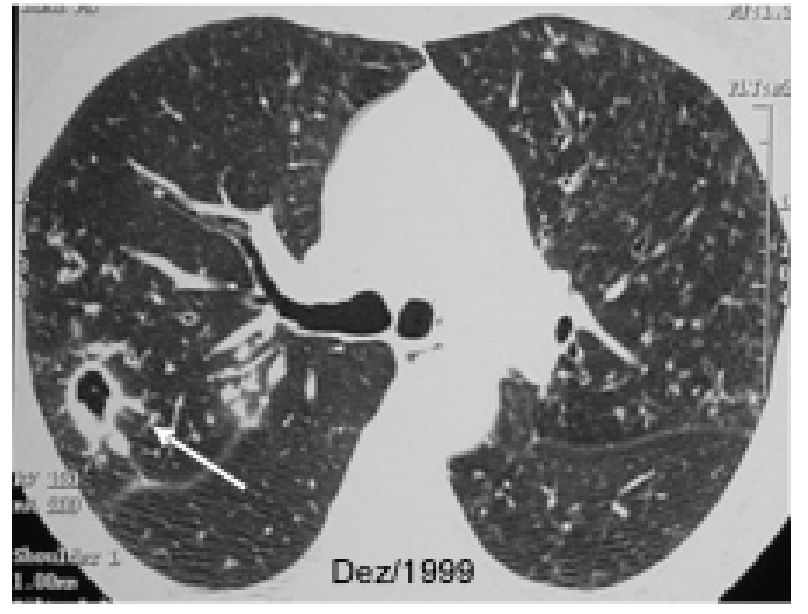




\section{Figure 7.}

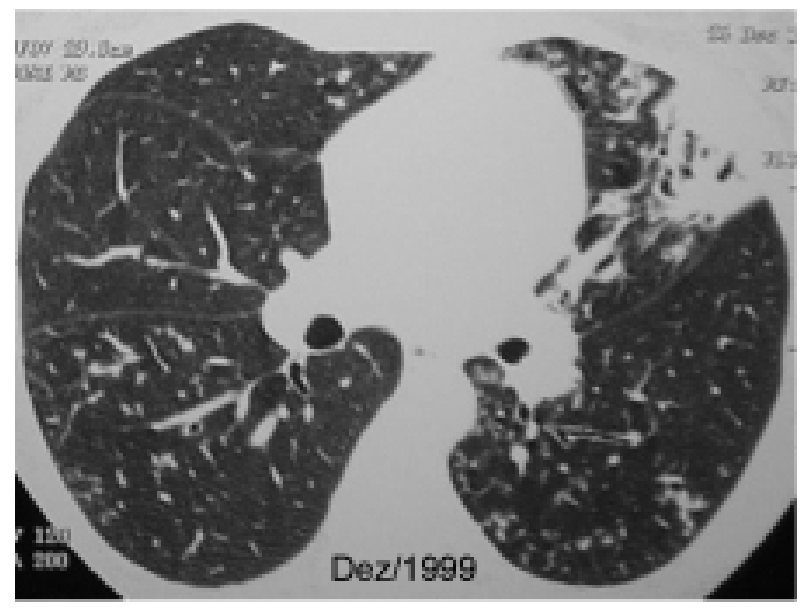

Figure 9.

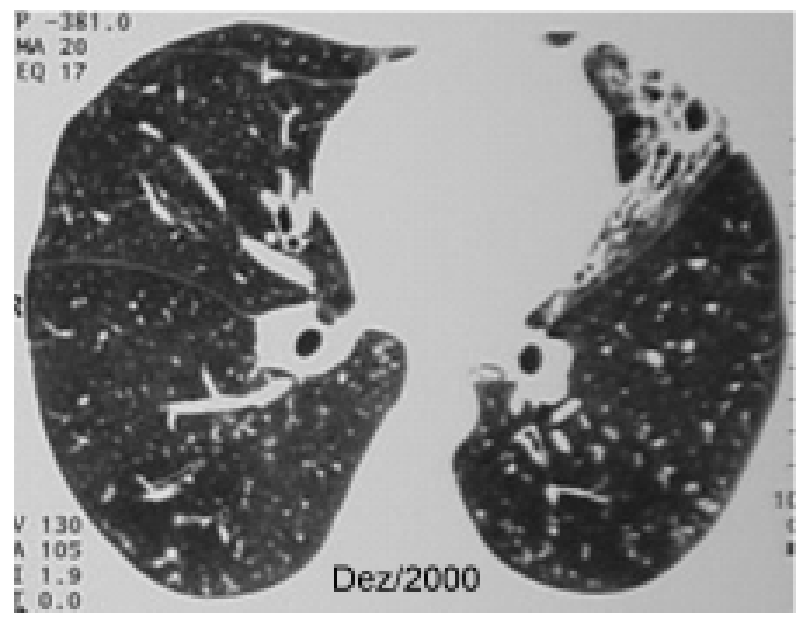

Figure 11.

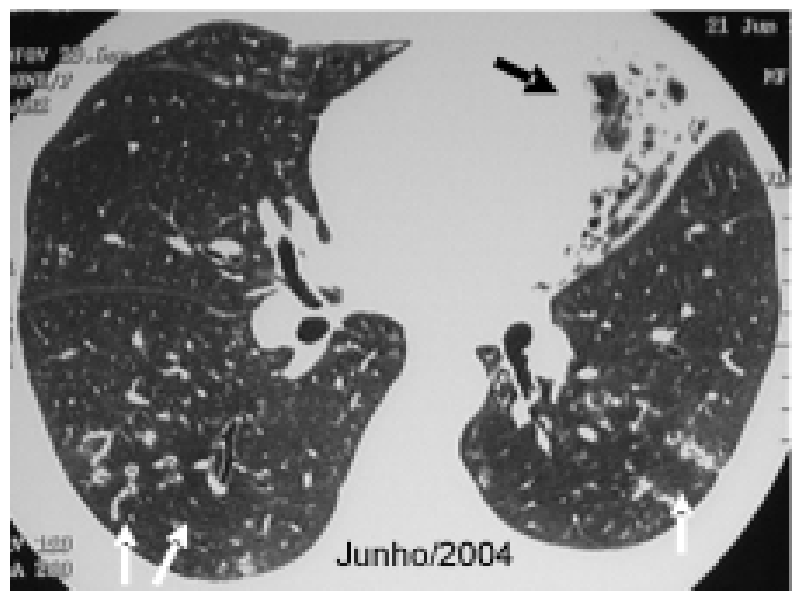

\section{Figure 8.}

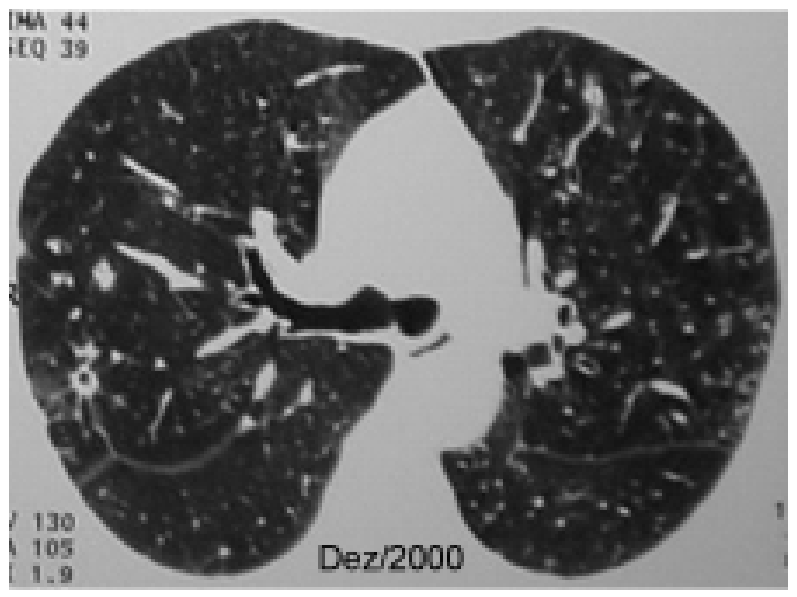

Figure 10.

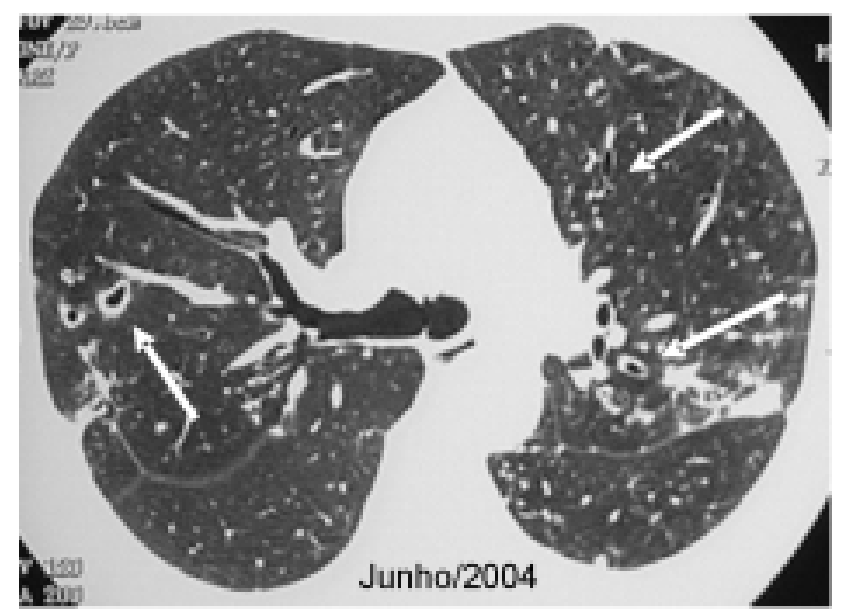

Figure 12.

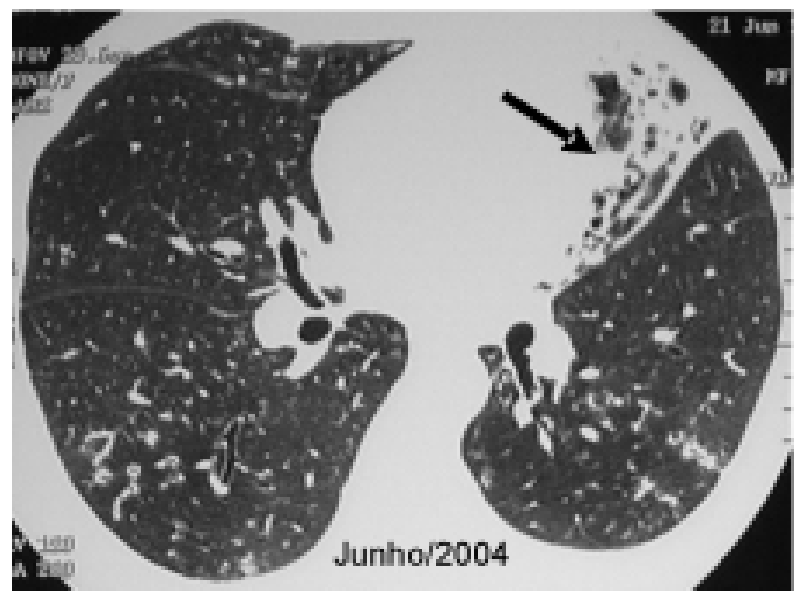


in the pathogenesis of MAC infection; this interaction can lead to granuloma formation, successful control of the infection, or clinical disease [8]. MAC pulmonary disease, which presents in a fibronodular form, has been described in individuals with scoliosis, pectus excavatum, mitral valve prolapse and joint hypermobility. These phenotypic abnormalities may be a form fruste of a yet-unidentified connective tissue disease. The mechanisms involved are not defined, but poor secretion drainage, or ineffective mucociliary clearance, are possible explanations $[15,16]$.

MAC can induce four distinct clinical syndromes: pulmonary disease, lymphadenitis, disseminated disease and, rarely, skin disease [1].

Patients with pulmonary disease related to NTM, especially MAC, can be divided into four groups:

a) The typical form has been described in elderly white men $[1,2,5]$ with previous pulmonary disease (for example, tuberculosis complications, active tuberculosis, bronchiectasis, pneumoconiosis, chronic aspiration pneumonia, bronchogenic carcinoma, and COPD) [1-6]. Radiographic patterns are characterized by nodules and by consolidation or cavities, most often affecting upper lobes and upper segments of lower lobes; these patterns may be indistinguishable from pulmonary tuberculosis $[4,6,14]$. Smoking and alcoholism are also risk factors [8].

b) Immunocompetent nonsmoking women over age 50 without underlying lung disease $[5,8,14]$. The typical radiographic patterns are bronchiectasis and small nodules in the mid lung field. Fever may not occur. The mean duration of symptoms is 25 weeks. [2].

c) Cystic fibrosis carriers [17].

d) Patients with immunosupression (ex: AIDS) $[2,3]$.

MAC pulmonary disease can occur in patients with or without previous pulmonary disease. The clinical manifestations are variable and are not specific. They include, in order of frequency, chronic cough (productive or dry), fatigue, dyspnea, hemoptysis, weakness and chest discomfort $[1,8]$. Fever and weight loss occur less frequently than in patients with typical tuberculosis [8]. All these manifestations can be confused with signs and symptoms of a previous pulmonary disease $[1,8]$. These symptoms are also observed in patients with tuberculosis, and the finding of a positive AFB smear indicates treatment for tuberculosis. Our patient was treated for three years as a tuberculosis carrier, initially with no previous treatment, and subsequently as resistant to the first and second (IR) regimens; she was then submitted to the third (III) regimen. She was finally considered to be a multiresistant tuberculosis carrier and was conducted to a specialized service. At this point, the type of mycobacteria was identified for the first time. At the MRTB outpatient clinic of the 'Octávio Mangabeira' Specialized Hospital (HEOM), among 231 patients with available culture results, Mycobacterium tuberculosis was isolated from $212(91.8 \%)$ and NTM from $19(8.2 \%)$ [18]. Therefore, the typing of mycobacteria is necessary, especially in patients with no previous treatment, who do not respond to the first regimen, despite the correct use of medications; this is considered primary resistance. In our patient, the slight improvement in imaging exams observed after the third regimen could be explained by the effect that some drugs in this regimen have on MAC.

There are differences between lesions caused by NTM and $M$. tuberculosis in chest roentgenograms. Both can cause cavities, but in NTM infections the cavities have thinner walls and there is less surrounding parenchymal opacity. There is less bronchogenic spread of disease, and pleural effusions are uncommon, but reactive pleural thickening can be seen. Solitary nodules without necrosis, reticulonodular infiltrates and dense consolidations have also been described $[1,19,20]$. The chest HRCTs of patients with MAC infections show that up to $90 \%$ of them have mid and lower lung field lesions. Multifocal bronchiectasis and clusters of small nodules $(<5 \mathrm{~mm})$, as seen in our patient, is a frequent finding $[1,7]$. The finding of this association is the most common presentation of MAC pulmonary infection in patients without predisposing conditions and/or 
immunosupression [7]. Our patient had HRCTs showing pulmonary lesions from June 1998 to June 2004; we observed that many of the nodules, especially in the middle and lower right lobes, became bronchiectasis. This fact was already documented in a case in which there was MAC granuloma previous to a lung nodule [21]. Pathological findings demonstrate that the lesions preceding bronchiectasis, in patients without previous pulmonary disease, begin as MAC granulomas, throughout the airway, which appear in the chest HRCT as nodular lesions [4].

The diagnosis of MAC disease is done essentially by isolation and identification of the microorganism in cultures. Nevertheless, one positive culture is insufficient, because the microorganism is frequently found in nature; a single culture could mean either colonization or infection. This differentiation between colonization and infection is very important. The precise diagnosis of MAC pulmonary disease is done following the criteria for the diagnosis of NTM [1]. One of the following items is necessary:

a) In at least three sputum/bronchial wash samples available within one year:

- Three positive cultures with a negative AFB smear result or

- Two positive cultures and one positive AFB smear result.

b) One bronchial wash and unavailability of sputum samples:

- Positive culture with $2+, 3+$, or 4+ growth on solid media or

- Positive culture with $2+, 3+$, or 4+ AFB smear.

c) Tissue biopsy

- Transbronchial or lung biopsy yielding an NTM

- Granulomatous inflammation with at least one sputum or bronchial wash positive for NTM.

Patients with cavitary disease easily present a positive AFB smear. On the other hand, nodular disease presents few organisms, with a negative or intermittently positive AFB smear [8].
Considering the bronchiectasis, it has been questioned whether MAC is the cause or if the existence of bronchiectasis is a predisposing condition for MAC pulmonary infection. There are studies indicating cases of MAC as a cause of bronchiectasis, especially in women over age 40 without predisposing conditions [4,5,7], and in other studies MAC has been documented in patients with bronchiectasis, these being cystic fibrosis carriers, or not.

The role of MAC in this situation is still unclear, especially in cystic fibrosis, where it can be present in lower airways for years without clinical and/or functional deterioration. Comparing cystic fibrosis carriers with and without NTM, the NTM positives (72\% MAC) were significantly more likely to be older, had a higher forced expiratory volume in one second (VEF1), had $S$. aureaus isolated, and $P$ aeruginosa was less frequent [14]. In cystic fibrosis, the decision to treat MAC is based on the finding of more than one positive culture, with a decline in clinical and radiographic features [22].

The optimal therapeutic regimen has yet to be established. Therapy should consist of a minimum three-drug regimen of clarithromycin, rifampin and ethambutol, until the patient is culture-negative after therapy for one year. Intermittent streptomycin for the first two to three months of therapy may be considered for extensive disease conditions. There are other drugs that can be used in the treatment, such as quinolones, clofazimine, cycloserine and ethionamide [1]. Another treatment proposal is to use clarithromycin or azithromycin, ethambutol and clofazimine to treat patients for a minimum of 12 months [23]. The studies published since 1994 have included a macrolide, either clarithromycin or azithromycin, usually in combination with ethambutol and a rifamycin [24].

We call special attention to the possibility of MAC being a cause of bronchiectasis, as has been mentioned in the literature, and we emphasize the necessity of identifying the type of mycobacteria in special situations, such as: a) persistence of a positive AFB smear, despite a correctly conducted first treatment for tuberculosis; b) unsuccessful III regimen; and c) MRTB carriers. 


\section{Acknowledgements}

This study was carried out by the Octávio Mangabeira Specialized Hospital (HEOM) Pneumology Research Center (NUPEP), Health office of Bahia (SESAB). It was supported by the Baiana Association to help Tuberculosis Control (ABACONNT).

\section{References}

1. ATS. Diagnosis and treatment of disease caused by nontuberculous mycobacteria. Am J Respir Crit Care Med 1997; 156:S1-S25.

2. Prince D.S., Peterson D.D., Steiner R.M. Infection with Mycobacterium avium complex in patients without predisposing conditions. N Engl J Med 1989;321:863-8.

3. Watanabe K.M.F., Kasahara K. Characteristics of pulmonary Mycobacterium avium-intracellulare complex (MAC) infection in comparison with those of tuberculosis. Respir Med 2003;97:654-9.

4. Fujita J., Ohtsuki Y., Shigeto E. Pathological findings of bronchiectases caused by Mycobacterium avium intracellulare complex. Respir Med 2003;97:933-8.

5. Reich J.M., Johnson R.E. Mycobacterium avium complex pulmonary disease presenting as an isolated lingular or middle lobe pattern. The Lady Windermere syndrome. Chest 1992;101:1605-9.

6. Fujita J. Clinical features of eleven cases of Mycobacterium avium-intracellulare complex pulmonary disease associated with pneumoconiosis. Respir Med 2004.

7. Swensen S.J., Hartman T.E., Williams D.E. Computed tomographic diagnosis of Mycobacterium aviumintracellulare complex in patients with bronchiectasis. Chest 1994; 105:49-52.

8. Griffith D.E., Richard Wallace R.J. Nontuberculous Mycobacterial Infections, Up to Date, 2003.

9. Wolinsky E. Nontuberculous mycobacteria and associated diseases. Am Rev Respir Dis 1979;119:107-59.

10. Meissner G., Anz W. Sources of Mycobacterium avium complex infection resulting in human diseases. Am Rev Respir Dis 1977;116:1057-64.

11. Meissner P.S., Falkinham J.O. Plasmid DNA profiles as epidemiological markers for clinical and environmental isolates of Mycobacterium avium, Mycobacterium intracellulare, and Mycobacterium scrofulaceum. J Infect Dis 1986; 153:325-31.

12. Von Reyn C.F., Maslow J.N., Barber T.W. Persistent colonisation of potable water as a source of Mycobacterium avium infection in AIDS. Lancet 1994;343:1137-41.
13. Du Moulin G.C., Stottmeier K.D., Pelletier P.A. Concentration of Mycobacterium avium by hospital hot water systems. JAMA 1988;260:1599-601.

14. Olivier K.N., Weber D.J., Wallace R.J. Nontuberculous mycobacteria. I: multicenter prevalence study in cystic fibrosis. Am J Respir Crit Care Med 2003; 167:828-34.

15. Huang J.H., Kao P.N., Adi V. Mycobacterium aviumintracellulare pulmonary infection in HIV-negative patients without preexisting lung disease: diagnostic and management limitations. Chest 1999; 115:1033-40.

16. Iseman M.D., Buschman D.L., Ackerson L.M. Pectus excavatum and scoliosis. Thoracic anomalies associated with pulmonary disease caused by Mycobacterium avium complex. Am Rev Respir Dis 1991;144:914-6.

17. Kilby J.M., Gilligan P.H., Yankaskas J.R. Nontuberculous mycobacteria in adult patients with cystic fibrosis. Chest 1992; 102:70-5.

18. Matos E.D., Lemos A.C.M. Nontuberculous mycobacteria at a multiresistant tuberculosis reference center in Bahia: clinical and epidemiological aspects. Braz J Infect Dis. In press. 2004.

19. Woodring J.H., Vandiviere H.M. Pulmonary disease caused by nontuberculous mycobacteria. J Thorac Imaging 1990;5:64-76.

20. Christensen E.E., Dietz G.W., Ahn C.H. Initial roentgenographic manifestations of pulmonary Mycobacterium tuberculosis, $M$ kansasii, and $M$ intracellularis infections. Chest 1981;80:132-6.

21. Moore E.H. Atypical mycobacterial infection in the lung: CT appearance. Radiology 1993; $187: 777-782$

22. Olivier K.N., Weber D.J., Lee J.H. Nontuberculous mycobacteria. II: nested-cohort study of impact on cystic fibrosis lung disease. Am J Respir Crit Care Med 2003; $167: 835-40$.

23. Stephen K. Field, Robert L. Cowie. Treatment of Mycobacterium avium intracellulare complex Lung Disease With a macrolide, ethambutol, and clofazimine Chest 2003; 124:1482-6.

24. Stephen K. Field, Dina Fisher, Robert L. Cowie. Mycobacterium avium complex. Pulmonary Disease in Patients Without HIV Infection. Chest 2004;126:566-81. 